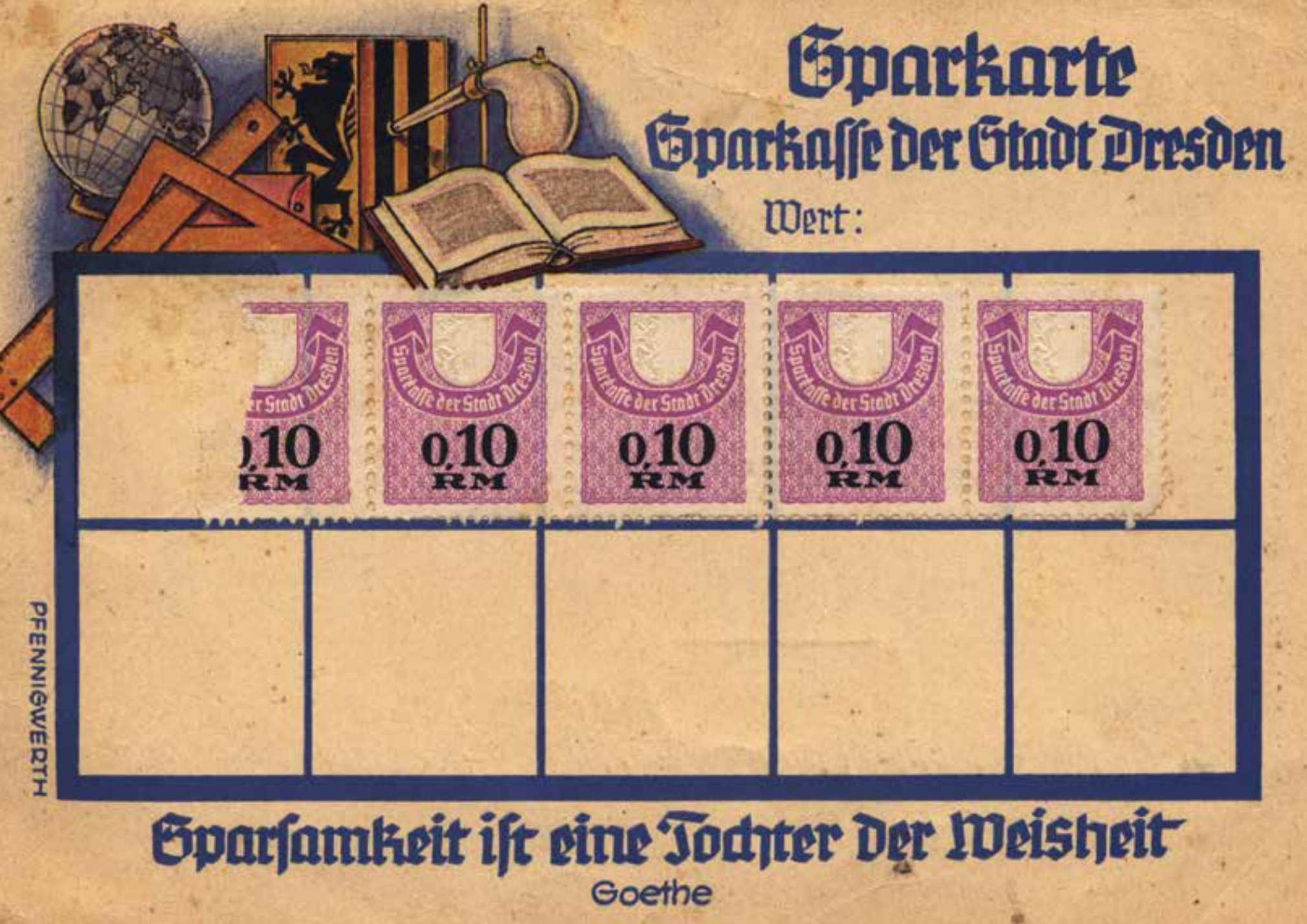

Während der NS-Zeit wurde in der Schule vor allem mit Sparmarken und Sparkarten gespart. - Historisches Archiv des Ostdeutschen Sparkassenverbandes

\section{Die sächsische Sparkassen- und Giroorganisation im Nationalsozialismus}

1 Vgl. Zeitschrift des K. Sächsischen Statistischen Landesamtes 58 (1912), S. 370 f. Als erste Verbandssparkasse hat wohl die 1843 im Gerichtsbezirk Schönfeld bei Dresden gegründete zu gelten, da für diese Einrichtung auch gleich eine Garantie der teilnehmenden Gemeinden bestand. Vgl. Gesetz- und Verordnungsblatt für das Königreich Sachsen, 1843, 18. Stück, S. 250.
Sparkassen gibt es in Sachsen seit 1819. In Zittau entstand 1825 die erste Einrichtung in kommunaler Trägerschaft. Dieses Modell setzte sich im Laufe der Jahre durch. Zahlreiche Sparkassen wurden von Stadt- und Landgemeinden ins Leben gerufen und private kommunalisiert. 1843 wurde die erste Verbandssparkasse gegründet. Solche Sparkassen in gemeinsamer Trägerschaft benachbarter $\mathrm{Ge}$ meinden gab es vermehrt ab dem ausgehenden 19. Jahrhundert. ${ }^{1}$ Seit 1907 hatten die kommunalen Sparkassen im Sächsischen Sparkassenverband eine dauerhafte politische Interessenvertretung. Der Verbandsvorsteher Dr. Johann Christian Eberle (1869-1937) war maßgeblich an der Gründung des Giroverbandes Sächsischer Gemeinden 1908 beteiligt und engagierte sich für die Verbreitung des bargeldlosen Zahlungsverkehrs durch die Sparkassen im Deutschen Reich. Im Gegensatz zum übrigen Deutschland waren in Sachsen jedoch nicht die Sparkassen, sondern selbstständige Girokassen für den Überweisungsverkehr zuständig. ${ }^{2}$ Eine weitere Besonderheit des Landes stellte die enorme Dezentralisierung dar. So existierten vor der Machtübernahme der Nationalsozialisten 355 kommunale Sparkassen im Freistaat. Im Giroverband waren 531 Gemeinden Mitglied. Es handelte sich überwiegend um kleine Einrichtungen. Erst 1943 erfolgte eine Anpassung der strukturellen Verhältnisse an die in den anderen Teilen des Großdeutschen Reichs durch den sächsischen Reichsstatthalter auf Anordnung des Reichswirtschaftsministers. ${ }^{3}$ Diese Neuordnung wird im vor- 
liegenden Aufsatz nicht behandelt. Es werden vielmehr drei ausgewählte Themenbereiche betrachtet. Die Einbindung der Sparkassen- und Giroorganisation in das NS-System wird so beleuchtet. Grundlage sind neben Literatur die im Historischen Archiv des Ostdeutschen Sparkassenverbandes noch vorhandenen Unterlagen der ehemaligen Verbandsorganisation, etwa Geschäftsberichte und Rundschreiben.

\section{Die Gleichschaltung der \\ Verwaltung und des Personals}

Gemäß der Sächsischen Sparkassen- und Girokassenverordnung vom 12. Mai 1932 wurden die Geldinstitute vom Verwaltungsrat geführt, in dem der Bürgermeister den Vorsitz hatte. Weitere Mitglieder waren ausgewählte Gemeinderäte und -verordnete sowie von ihnen gewählte Bürger, die nicht der Kommunalverwaltung angehörten. ${ }^{4}$ Das Personal der Kassen bestand aus Beamten, Angestellten und Arbeitern, welche die Gewährträger bereitstellten. Im Zuge der „Machtergreifung“ in Sachsen nach den Reichstagswahlen vom 5. März 1933 wurden nicht nur von Ortsgruppen- und Kreisleitern der NSDAP sowie SA-Führern, sondern auch durch den am 8. März 1933 eingesetzten Reichskommissar Manfred Freiherr von Killinger (1886-1944) politische Säuberungen vorgenommen. ${ }^{5}$ Das Vorgehen auf kommunaler Ebene war schwer zu kontrollieren. Der Reichskommissar musste sogar anweisen, dass nicht eigenmächtig in die Geschäfte der Spar- und Girokassen einzugreifen sei und keine Überprüfungen von Konten politischer Gegner stattzufinden hätten. ${ }^{6}$ Lokale Parteistellen versuchten, ihren Machtanspruch durchzusetzen. Es kam zu willkürlichen Beurlaubungen von Bürgermeistern und von kommunalen Beschäftigten.

Das sogenannte Berufsbeamtengesetz vom 7. April 1933 führte die Personalhoheit wieder in den staatlichen Bereich zurück und regelte nachträglich die Entlassung zuvor Beurlaubter rechtlich. ${ }^{7}$ „Es war das erste Gesetz des Dritten Reiches, welches unverdeckt offenes Sonderrecht gegen rassisch und politisch Missliebige setzte. ${ }^{8}$ Betroffen waren nicht nur Beamte, sondern auch Angestellte und Arbeiter. Es galt zudem für Bürgermeister als Wahlbeamte. Im Juni 1933 begann in Sachsen eine umfassende Überprüfungsaktion mit Fragebögen auch im kommunalen Bereich. ${ }^{9}$ Auf Vorschlag des Innenministeriums, das von seinem Gefolgsmann Karl Fritsch (1901-1944) geleitet wurde, verkündete der Reichsstatthalter und Gauleiter Martin Mutschmann (1879-1947) Entlassungen und Versetzungen in den Ruhestand. Erwähnt werden muss auch die Gleichschaltung der Gemeindevertretungen, die auf Grundlage reichsrechtlicher Bestimmungen erfolgte und zur Ausschließung kommunistischer und sozialdemokratischer Abgeordneter führte. Am 6. April wies der Reichskommissar die Auflösung der Verwaltungsräte der Sparkassen und Girokassen an. Erst nach der neu-

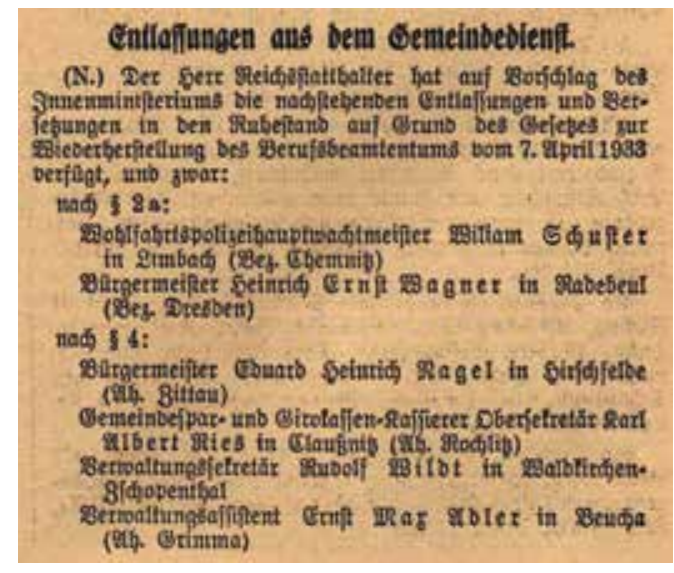

Im „Sächsischen Verwaltungsblatt" wurden, etwa am 29. September 1933, Entlassungen verkündet. ( ) Historisches Archiv des Ostdeutschen Sparkassenverbandes

en Zusammensetzung der Verordneten der Stadtbeziehungsweise Landgemeinden konnte die Neuwahl der Verwaltungsräte erfolgen. ${ }^{10}$

Die Veränderung der kommunalen Kollegien im Sinne der Nationalsozialisten hatte entsprechende Auswirkungen. Die dargestellte Personalunion war entscheidend bei der Gleichschaltung der Geldinstitute, die ab 1933 nicht nur in Sachsen unter dominierenden nationalsozialistischen Einfluss kamen. ${ }^{11}$ Die rechtliche Selbstständigkeit, die die sächsischen Sparkassen und Girokassen erst seit 1932 als Körperschaften öffentlichen Rechts besaßen, wurde durch die politische Besetzung der Verwaltungsämter schlichtweg negiert. ${ }^{12}$ Bald galt das „Führerprinzip“ in den Kommunalverwaltungen. Die Verwaltungsratsmitglieder der Kassen wurden ab 1935 nicht mehr gewählt, sondern vom Leiter der Gewährgemeinde als Vorsitzenden bestellt. ${ }^{13}$ Eine hohe Quote von Parteimitgliedern beim Personal wurde nach dem Ende der NS-Herrschaft mit der direkten Unterstellung unter die gemeindlichen Verwaltungen und mit dem Druck auf die Mitarbeiterschaft erklärt. ${ }^{14}$ Für die Beamten galten ab 1937 die Vorschriften des Deutschen Beamtengesetzes, das sie zur NS-Weltanschauung und zur „Führertreue“ verpflichtete.

Aber nicht nur die kommunalen Geldinstitute allein, auch ihre Verbände wurden 1933 personell umgestaltet. Gemäß sächsischen Gleichschaltungsgesetzen erledigten sich die Mandate der Vorstandsmitglieder. ${ }^{15}$ In Absprache mit dem Innenministerium und der Gauleitung der NSDAP wurde die Satzung geändert, ein gemeinsamer Vorstand für den Sparkassen- und den Giroverband gebildet, den Vertretern der Großstädte automatisch ein Sitz gesichert und ein Beauftragter des Gauleiters zum Stellvertreter Eberles gemacht. ${ }^{16}$ Curt Robert Lahr (1898-1974) hieß der Mann, welcher der Partei seit dem 1. August 1930 als Ortsgruppenleiter, dann Bezirksleiter und ab Mai 1933 als Gauamtsleiter angehörte. ${ }^{17} \mathrm{Er}$ war im Spar- und Girokassenwesen erfahren und schon lange mit dem Verbandschef bekannt. Unter anderem dieser wichtige Kontakt half Eberle, der sich zwar zur neuen Regierung bekannte, aber selbst nicht in die NSDAP eintrat, im Amt zu bleiben. ${ }^{18}$ Im Auftrag Mutschmanns legitimierte Lahr Dr. Jo-
2 Vgl. Barbara Hillen: Der Sparkassenreformer und sächsische Mittelstandspolitiker Johann Christian Eberle (1869 -1937). Beucha 2004, S. 126 f.

3 Vgl. Georg Andreas Handschuh: Der öffentliche Auftrag der sächsischen Sparkassen. Von der Inpflichtnahme Privater über die Reglementierung als öffentliche Aufgabe bis zur Geschäftstätigkeit nach eigentümerorientierten Oberzielen. Baden-Baden 2010, S. 113

4 Vgl. Sparkassen- und Girokassenverordnung vom 12 Mai 1932, § 7, OSV Archiv.

5 Vgl. Andreas Wagner: „Machtergreifung" in Sachsen. NSDAP und staatliche Verwaltung 1930-1945. Köln 2004, S. 136 ff.

6 Vgl. Verbandsrundschreiben vom 13. April 1933, OSV Archiv.

7 Vgl. Mike Schmeitzner/Andreas Wagner: Zweierlei „Machtergreifung“. Der diktatorische Zugriff von NSDAP und KPD/SED auf die sächsische Verwaltung. In: Günther Heydemann/Heinrich Oberreuther (Hrsg.): Diktaturen in Deutschland - Vergleichsaspekte. Strukturen, Institutionen und Verhaltensweisen. Berlin 2003, S. 64.

8 Bernhard Müller: Alltag im Zivilisationsbruch. Das Ausnahme-Unrecht gegen die jüdische Bevölkerung in Deutschland 1933-1945. München 2003, S. 65 .

9 Vgl. Wagner 2004 (wie Anm. 5), S. 227.

10 Vgl. Verbandsrundschreiben vom 6. Mai 1933, OSV Archiv.

11 Vgl. Hans Pohl/Bernd Rudolph/Günther Schulz: Wirtschafts- und Sozialgeschichte der deutschen Sparkassen im 20. Jahrhundert. Stuttgart 2005, S. $170 \mathrm{f}$.

12 Vgl. Handschuh 2010 (wie Anm. 3), S. 112.

13 Vgl. Zweite Verordnung zur Änderung der Sparkassenund Girokassenverordnung vom 27. September 1935 , $\S 7$, OSV Archiv. 
Zur Feier des 25. Geburtstags des Giroverbandes

Sächsischer Gemeinden am 5. Oktober $1933 \mathrm{im}$ Leipziger Zentraltheater waren nicht nur Bürgermeister als Vorsitzende der gleichgeschalteten

Verwaltungsräte eingeladen. ๑ Historisches Archiv des

Ostdeutschen Sparkassenverbandes

14 Vgl. Schreiben des Sächsischen Sparkassenverbandes an die Landesregierung Sachsen Ministerium des Innern vom 9. Januar 1947, OSV Archiv.

15 Vgl. Geschäftsbericht des Sächsischen Sparkassenverbandes für 1933, S. 3, OSV Archiv.

16 Vgl. Verbandsrundschreiben vom 8. August 1933 sowie vom 26. August1933 (Niederschrift über die Verbandsversammlung am 21. August 1933), OSV Archiv.

17 Die SS-Akte Lahrs im Bundesarchiv Berlin belegt nicht nur seine Parteitätigkeit. Er wurde 1937 nichthauptamtlicher Sturmbannführer und rückte bis zum Standartenführer auf.

18 Vgl. Hillen 2004 (wie Anm. 2), S. 197.

19 In der NSDAP-Mitgliederkartei im Bundesarchiv Berlin ist er erfasst.

20 Auf der Verbandsversammlung zur Gleichschaltung am 21. August 1933 hielten die Ehrengäste Ministerpräsident Manfred von Killinger und Innenminister Karl Fritsch aufschlussreiche Reden.

21 Verbandsrundschreiben vom 6. September 1933, OSV Archiv.

22 Vgl. Geschäftsbericht des Sächsischen Sparkassenverbandes für 1933, S. 3, OSV Archiv.

23 Vgl. Pohl/Rudolph/Schulz 2005 (wie Anm. 11), S. 176 f

24 Verbandsrundschreiben vom 7. Juni 1933, OSV Archiv.

25 Verbandsrundschreiben vom 19. Oktober 1933, OSV Archiv.

26 Vgl. Geschäftsbericht des Sächsischen Sparkassenverbandes für 1934, S. 8, OSV Archiv.

27 Vgl. Verbandsrundschreiben vom 29. Oktober 1936, OSV Archiv

28 Vgl. Geschäftsbericht des Sächsischen Sparkassenverbandes für 1938, S. 10, OSV Archiv.

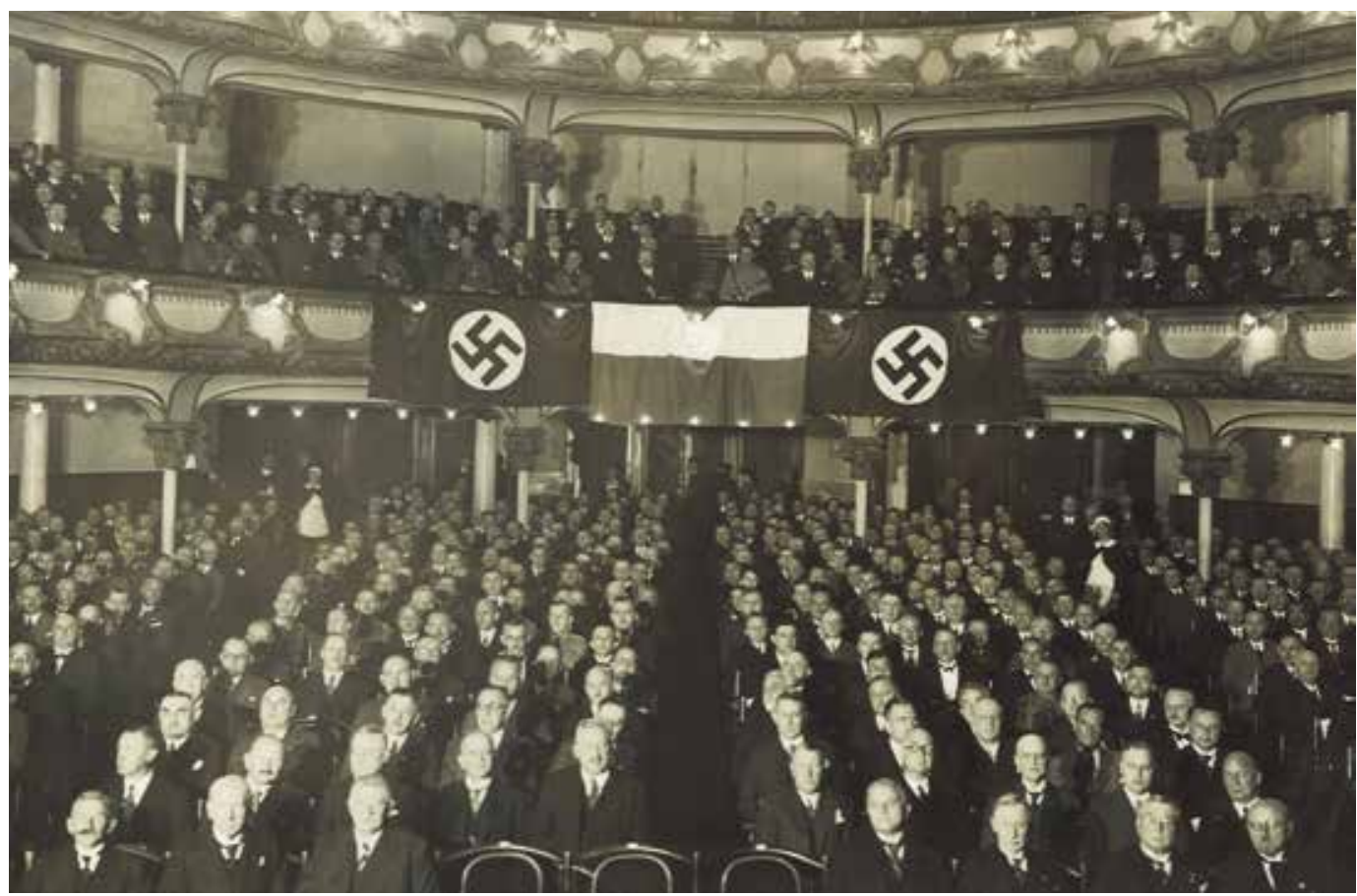

hann Christian Eberle als „Führer“ der Verbände. Als er 1938 mit Genehmigung des Reichswirtschaftsministers und des Gauleiters dessen Nachfolge antrat, war er bereits Ministerialdirektor und Leiter der sächsischen Staatskanzlei. Sein stellvertretender Verbandsvorsteher wurde zunächst Walter Dönicke (1899-1945), Oberbürgermeister von Leipzig, und nach dessen Absetzung der Oberbürgermeister von Chemnitz, Walter Schmidt (1903-1962). Beide waren „alte Kämpfer“ und NS-Funktionäre. Als zweiter Stellvertreter fungierte ab 1938 Dr. Oskar Gebauer, Bürgermeister von Kamenz, der erst am 1. Mai 1933 in die Partei eingetreten war. ${ }^{19}$

\section{Das Sparen vor dem und im Zweiten Weltkrieg}

Sparkassen als kommunale Geldinstitute mit gemeinnützigem Charakter galten den Nationalsozialisten als dem Wohl der von ihnen definierten „Volksgemeinschaft" verpflichtet. Auch sächsische NS-Größen fanden 1933 lobende Worte für ihr Wirken..$^{20}$ Der Sparkassenverband empfahl seinerseits den Mitgliedsinstituten, Maßnahmen zu ergreifen, um sich ,als Träger und Vermittler nationalsozialistischer Wirtschaftsgesinnung “21 darzustellen. Die traditionelle Gemeinwohlorientierung der regionalen und nicht in erste Linie auf Gewinn bedachten Geldinstitute wurde als ein Wert erkannt, der im Sinne Hitlers und des Nationalsozialismus sei. ${ }^{22}$ Die Förderung des Sparsinns war damals satzungsmäßig eine wichtige Aufgabe der Sparkassen. Die Regierung hatte großes Interesse daran, dass sich die Spareinlagen vermehrten. Darin wurde während der Weltwirtschaftskrise eine Grundvoraussetzung für das Ankurbeln der heimischen Wirtschaft und die Beseitigung der Arbeitslosigkeit gesehen. Der Staat wollte, dass jeder Ein- zelne sich für die Gemeinschaft einsetzte, seine Solidarität und Opferbereitschaft zeigte und eben nicht nur für seine privaten Zwecke, sondern auch zum Nutzen der Allgemeinheit sparte. ${ }^{23}$ „Gemeinnutz über Eigennutz" war die Devise. Dies schlug sich in der Sparkassenwerbung nieder. Der „Gedanke der Volksgemeinschaft, des Dienstes am Ganzen, der betonte Wille zum national-wirtschaftlichen Aufstieg [und] zur finanziellen Unabhängigkeit vom Ausland"24 sollten thematisiert werden. Und so wurde der Weltspartag Ende Oktober 1933 erstmals als Nationaler Spartag begangen. Die Parolen lauteten „Geldhamstern ist Sabotage am nationalen Aufbau“, „Der Sparpfennig schafft Arbeit und Brot" sowie „Sparen ist nationale Pflicht ${ }^{\text {"25. }}$.

Verschiedene Initiativen zur Forcierung des Kleinsparens liefen an, wobei meistens Sparmarken zum Einsatz kamen. Beispielsweise wurden auch die Sparkassen 1934 gemäß einer Vereinbarung mit der Deutschen Arbeitsfront Träger des Kraftdurch-Freude-Sparens. ${ }^{26}$ Gespart wurde für den Urlaub. Beim KdF-Sparen nahmen die Sachsen jahrelang eine reichsweite Spitzenposition ein. Die Sparkassen verkauften später auch Marken der DAF für den „Volkswagen“. 1936 wurde sie beim Zwecksparen der Hitler-Jugend aktiv. ${ }^{27}$ Hier ging es vorrangig um das Geldsammeln für Lagerfahrten. Dem schon länger verbreiteten Schulsparen wurde in der NS-Zeit große Aufmerksamkeit gewidmet, um der Jugend den Spargedanken als „nationale Pflicht" näher zu bringen. Natürlich ging es auch darum, neue Sparkassenkunden heranzuziehen. Auf das Engagement der Lehrerschaft, die etwa Sparmarken verkaufte, war man beim Schulsparen besonders angewiesen. Fördernd wirkte bei der Verbreitung unter anderem, dass sich der Reichsbildungsminister 1936 zum Pflichtsparen in 


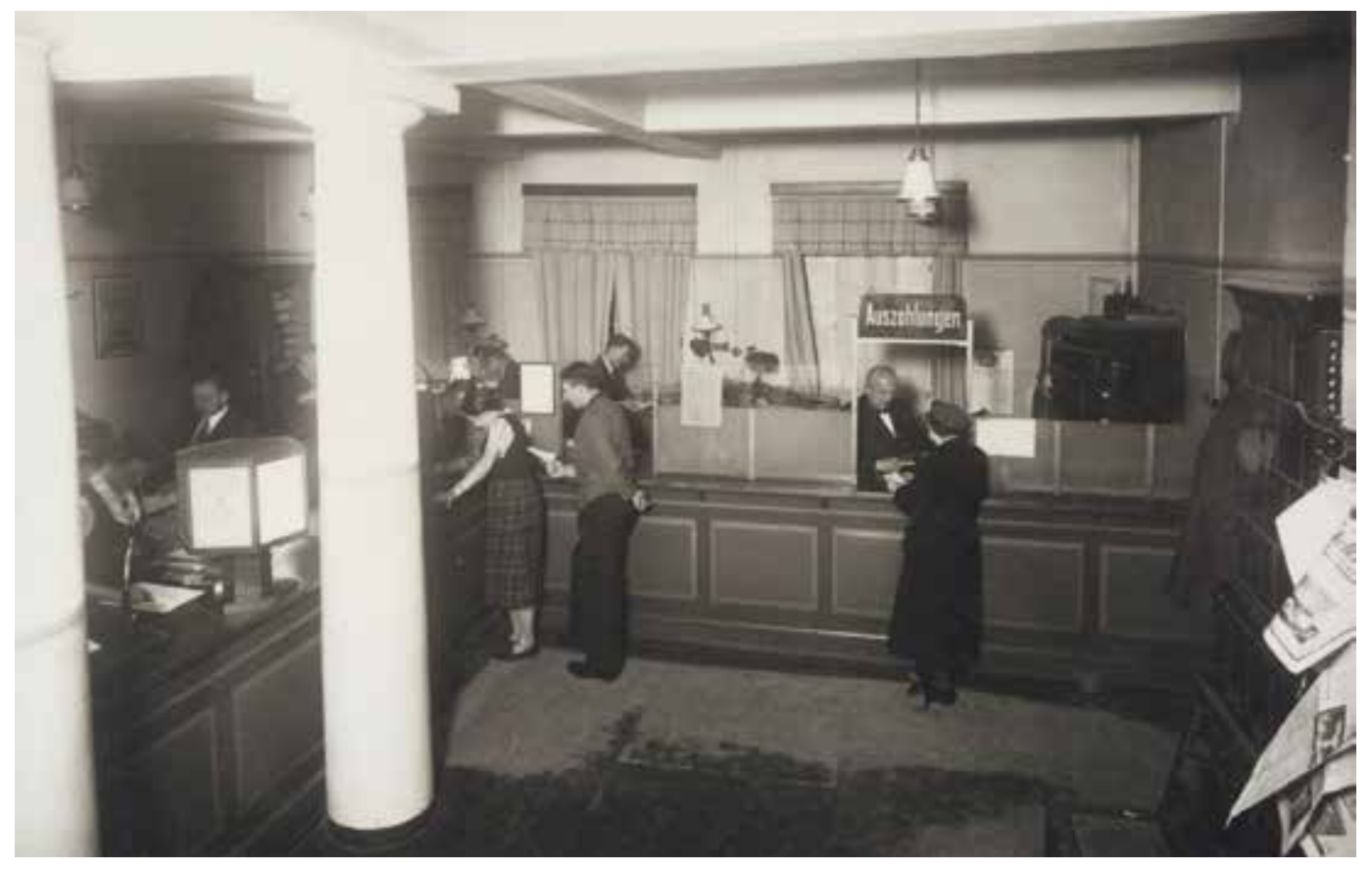

der Schule bekannte. Der Reichsstatthalter forderte 1938 sogar für jede Schule eine Schulsparkasse und für jeden Schüler ein Sparkassenbuch. ${ }^{28}$

Das Vertrauen der Bevölkerung zur NS-Führung und die politische Stabilität sowie die Verbesserung der Wirtschaftslage und die Arbeitsbeschaffungsmaßnahmen sind in den Geschäftsberichten des Sächsischen Sparkassenverbandes als Ursachen des Einlagenwachstums in den Friedensjahren genannt. Die Folgen des Krisenjahres 1931 im Spargeschäft wurden bereits 1933 überwunden, auch weil man der Reichsführung vertraut und wieder an eine stabile Währung geglaubt habe, so der Verband. ${ }^{29}$ Zudem konnte die Aufwertung und Erstattung der in der Inflation 1923 entwerteten Spareinlagen der Kunden endlich abgeschlossen werden. Hemmend wirkte sich jedoch auf das Einlagenwachstum aus, dass die Belebung der auf Export ausgerichteten sächsischen Industrie erst spät einsetzte und wegen der ausländischen Konkurrenz niedrige Löhne gezahlt wurden. Auch die Grenzlage Sachsens wurde als Hindernis für den wirtschaftlichen Wiederaufstieg gesehen. ${ }^{30}$ Die Annexion des Sudetenlandes 1938 beendete den Sonderstatus. Die politische Lage während der „Sudetenkrise“ führte allerdings zur Beunruhigung der Sparer, die mehr Geld abhoben als einzahlten. Intern war von Angstabhebungen die Rede. Auffällige Abhebungen führten sogar zu Nachforschungen durch lokale Parteistellen, die im Namen des Gauleiters unterbunden werden mussten. ${ }^{31}$ Auch beim Beginn des Zweiten Weltkrieges ist das Phänomen des Auszahlungsüberschusses festzustellen. Wieder schloss der Verband Angstabhebungen offiziell aus, präsentierte stattdessen als wichtigste Ursache die Versorgung der Einberufenen mit Bargeld. ${ }^{32}$ Die Kassen wurden indes angewiesen, auffällige Kunden über die ,volkswirtschaftliche Schädlichkeit und die Strafbarkeit des
Geldhamsterns“33 zu informieren. Auf keinen Fall sollte jedoch örtlichen Behörden oder NSDAPStellen die Möglichkeit gegeben werden, den Schalterverkehr $\mathrm{zu}$ überwachen oder $\mathrm{zu}$ kontrollieren. Der Reichswirtschaftsminister, der Reichspropagandaminister sowie der Reichsführer SS hätten dies untersagt. Im Krieg konnten Empfehlungen des Sparkassenpersonals am Kundenschalter, doch lieber nicht das Konto zu leeren, zu Gerüchten führen, die bekämpft wurden. ${ }^{34}$ So erfuhr die Kundschaft, dass nach dem „Heimtückegesetz" Strafen drohten, wenn jemand behauptete, ein Teil des Geldes werde für Bombengeschädigte einbehalten. ${ }^{35}$

Im Zeitraum von der Ernennung Hitlers zum Reichskanzler 1933 bis zum Ausbruch des Krieges 1939 haben sich die Spareinlagen in Sachsen von 750 Millionen auf 1,6 Milliarden Reichsmark mehr
Personal und Kundschaft in der Stadtsparkasse Borna, 1939

- Historisches Archiv des

Ostdeutschen Sparkassenverbandes

29 Tatsächlich setzte das NSRegime auf vertrauensbildende Maßnahmen, etwa indem Hitler Hjalmar Schacht als Reichsbankpräsidenten einsetzte. Vgl. Simone Reinhardt: Die Reichsbank in der Weimarer Republik. Eine Analyse der formalen und faktischen Unabhängigkeit. Frankfurt am Main/Berlin/ Bern/Bruxelles/New York/ Oxford/Wien 2000, S. 265 f.

30 Vgl. Geschäftsbericht des Sächsischen Sparkassenverbandes für 1938, S. 8 f., OSV Archiv. Zu den nachteiligen Wirkungen siehe Rainer Karlsch/Michael Schäfer: Wirtschaftsgeschichte Sachsens im Industriezeitalter. Leipzig 2006, S. 213.

31 Vgl. Verbandsrundschreiben vom 13. Oktober 1938, OSV Archiv.

32 Vgl. Geschäftsbericht des Sächsischen Sparkassenverbandes und des Giroverbandes Sächsischer Gemeinden für 1939, S. 4, OSV Archiv.

33 Verbandsrundschreiben vom 15. September 1939, OSV Archiv.

34 Vgl. Verbandsrundschreiben vom 17. September 1943, OSV Archiv.

Im Zweiten Weltkrieg vermehrten sich die Spareinlagen enorm. - Historisches Archiv des Ostdeutschen Sparkassenverbandes

\section{Spareinlagen am Jahresende in Mrd. RM}

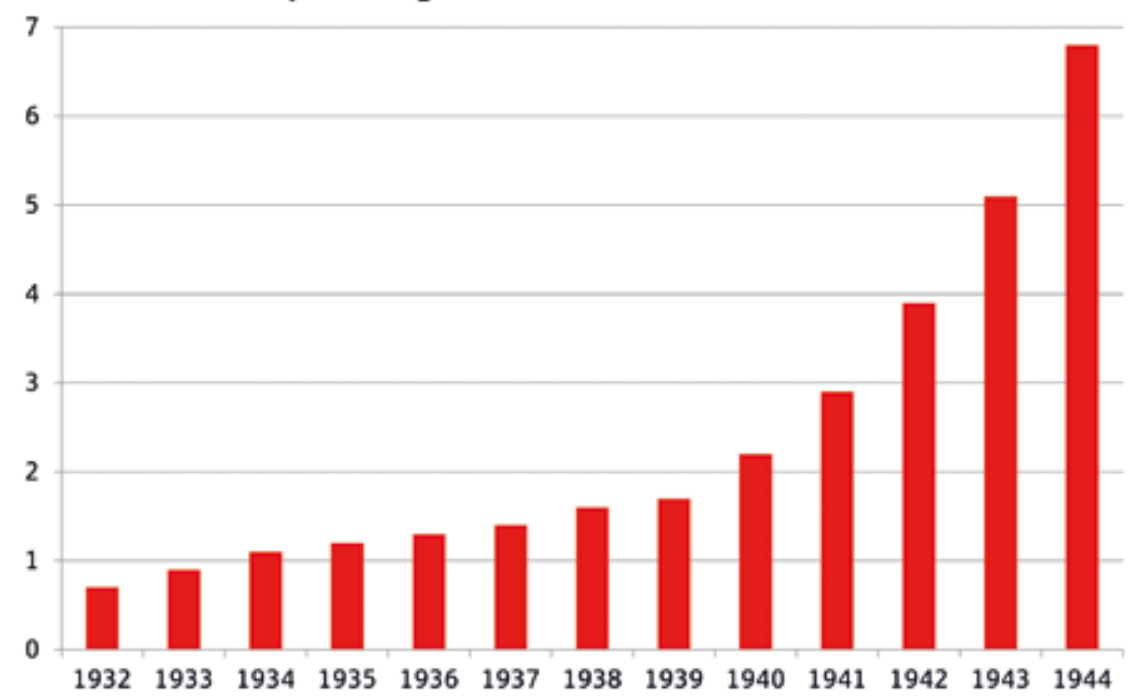


35 Durch Aushänge in den Geschäftsstellen sollten zwei Fälle von Bestrafungen sächsischer Sparkassenkunden publik gemacht werden. Vgl. Verbandsrundschreiben vom 4. Februar 1944 sowie vom 17. März 1944, OSV Archiv.

36 Vgl. Bericht des Sächsischen Sparkassenverbandes vom 10. August 1948, OSV Archiv.

37 Geschäftsbericht des Sächsischen Sparkassenverbandes und des Giroverbandes Sächsischer Gemeinden für 1939, S. 6, OSV Archiv.

38 Vgl. Geschäftsbericht des Sächsischen Sparkassenverbandes und des Giroverbandes Sächsischer Gemeinden für 1941, S. 12, OSV Archiv.

39 Vgl. Geschäftsbericht des Sächsischen Sparkassenverbandes und des Giroverbandes Sächsischer Gemeinden für 1942, S. 2, OSV Archiv.

40 Vgl. Verbandsrundschreiben vom 30. September 1942, OSV Archiv.

41 Vgl. Geschäftsbericht des Sächsischen Sparkassenverbandes und des Giroverbandes Sächsischer Gemeinden für 1942, S. 1, OSV Archiv.

42 Vgl. Geschäftsbericht des Sächsischen Sparkassen- und Giroverbandes für 1943, S. 2, OSV Archiv.

43 Vgl. Pohl/Rudolph/Schulz 2005 (wie Anm. 11), S. 196.

44 Vgl. Bernd Sprenger: Das Geld der Deutschen. Geldgeschichte Deutschlands von den Anfängen bis zur Gegenwart. Paderborn 2002, S. 228.

45 Geschäftsbericht des Sächsischen Sparkassen- und Giroverbandes für 1943, S. 3, OSV Archiv.

46 Vgl. Verbandsrundschreiben vom 26. Januar 1935, OSV Archiv.

47 Vgl. Geschäftsbericht des Sächsischen Sparkassenverbandes für 1936, S. 7 sowie für 1937, S. 14, OSV Archiv.

links: Auch beim Abholsparen kamen Klebemarken zum Einsatz, die den Sparbetrag belegten. (-) Historisches Archiv des Ostdeutschen Sparkassenverbandes

rechts: Titelbild eines Flugblatts, das "Geldhamstern" als Verrat am

Vaterland bezeichnete, 1940

( ) Historisches Archiv des

Ostdeutschen Sparkassenverbandes als verdoppelt. Die Zahl der Sparkonten vermehrte sich von 1,6 auf 4 Millionen. Bei Kriegsende sollten es 5,5 Millionen sein. Die Guthaben der Sparer wuchsen im Zweiten Weltkrieg auf 7,2 Milliarden Reichsmark. ${ }^{36}$ Sie haben sich also mehr als vervierfacht. Mit dem Krieg erlangte das seit 1938 praktizierte Gefolgschaftssparen, das gemeinschaftliche Sparen der Mitarbeiterschaft in den Betrieben, besondere Bedeutung. „Wenn es das Ziel der Reichsregierung sein muss, als eines der Mittel zur Finanzierung des Krieges die überschüssige Kaufkraft abzuschöpfen, dann tut das besonders wirksam das Gefolgschaftssparen, weil es die Kaufkraft an der Quelle abschöpft und in Sparkapital umwandelt.“" ${ }^{37}$ Zur Gewinnung von Spareinlagen wurde sogar auf das regelmäßige Abholen von Geldern durch „Sparpfleger“ gesetzt, damit der „Volksgenosse" nicht etwa sein Bares zu Hause hortete. Es gab aber auch Anreize zum Sparen. So führte die Reichsregierung 1941 das Eiserne Sparen ein, wobei ein Teil des Lohns und Gehalts steuerbefreit bis nach dem erhofften „Endsieg“ festgemacht wurde. Bei dieser währungspolitischen Maßnahme, die überschüssige Kaufkraft binden sollte, wirkten die sächsischen Sparkassen mit. ${ }^{38}$ Eine Losung in der Werbung lautete „Im Kriege sollst du eisern sparen - im Frieden kaufst du dafür Waren“. Der Erfolg war aber, auch weil die Steuervorteile den langfristigen Verzicht auf die jederzeitige Verfügung der Einlagen nicht wettmachten, mit 39,3 Millionen Reichsmark 1942 eher bescheiden. ${ }^{39}$

Mehr Geld wurde in dem Jahr allein während der Deutschen Sparwoche zur Sparkasse gebracht. Erstmals fand nicht nur ein Spartag, sondern eine ganze Sparwoche statt. Beworben wurde damals das „Heer der Sparer“, das seine privaten Gelder zur Nutzung durch die Kriegswirtschaft bereitstellen und so sein Vertrauen in den „Endsieg“" bewei-

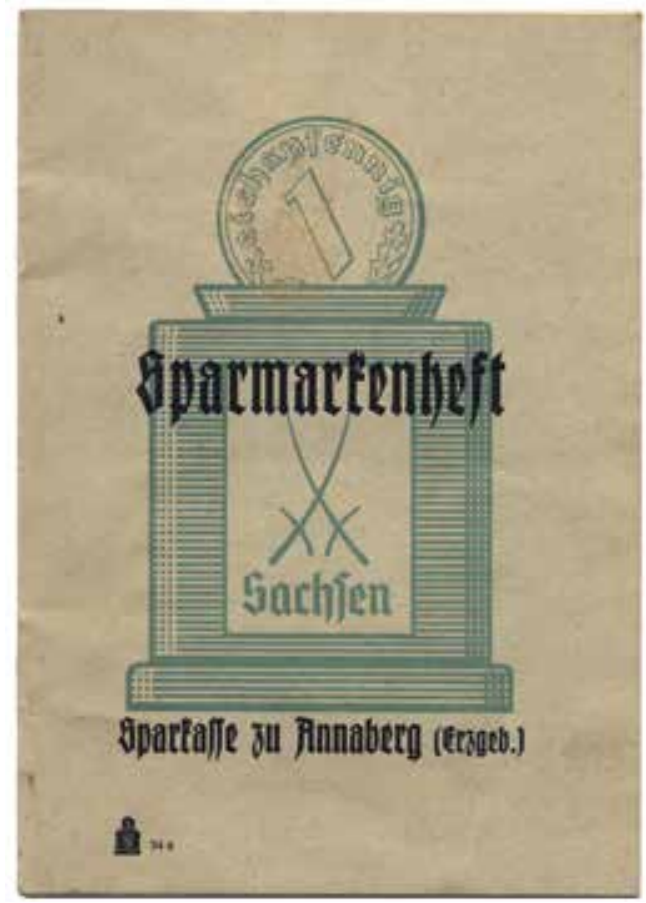

sen sollte. ${ }^{40}$ Dem Sparen an der „Heimatfront“ wurde kriegsentscheidende Bedeutung zugemessen. Auch Maßnahmen zur Sparförderung trugen dazu bei, dass 1942 mit einer Einlagensteigerung von 34,5 Prozent das Rekordjahr im Zweiten Weltkrieg wurde. So bestimmte etwa das Sächsische Ministerium für Volksbildung unter der Leitung von Arthur Göpfert (1902-1986) das Schulsparen zum verbindlichen Erziehungsmittel in allen Schulgattungen. ${ }^{41}$ Im Gegensatz zur Friedenszeit lagen die Sachsen nun beim Sparen über dem Reichsdurchschnitt, was der Verband auf ihre „Sparkraft“ und ihren „Sparwillen“ zurückführte. Bereits 1943 hatte statistisch gesehen jeder Einwohner des Landes ein Sparkassenbuch, auf dem durchschnittlich 950 Reichsmark lagen. ${ }^{42}$ Gespart wurde so viel, weil den Menschen aufgrund eingeschränkter privater Konsummöglichkeiten, etwa wegen Rationalisierungen, und des Mangels alternativer Anlagemöglichkeiten schlichtweg kaum eine andere Option blieb. ${ }^{43}$ Die Politik des Regimes führte mit einer enormen Vermehrung der Geldmenge zur Finanzierung der Kriegswirtschaft bei gleichzeitiger Kontrolle der Preise zu einer „zurückgestauten Inflation“. ${ }^{44}$ Durch die Forcierung des Sparens wurde wie im Ersten Weltkrieg überschüssiges Geld abgeschöpft.

Die enormen Einlagenzuwächse dienten dazu, den Bestand der sächsischen Sparkassen an Anleihen und Schatzanweisungen des Deutschen Reichs zu vermehren und so „einen sehr erheblichen Beitrag zur Kriegsfinanzierung“45 zu leisten. Teile des Volkseinkommens wurden vom Staat als Kredit genommen und für seine verbrecherischen Ziele eingesetzt. Bereits seit 1934 erfolgte eine zunehmende Anlage in Wertpapieren des Reichs. Ab 1935 konnten diese sogar teilweise auf die Liquiditätsreserven angerechnet werden. ${ }^{46}$ So wollte der

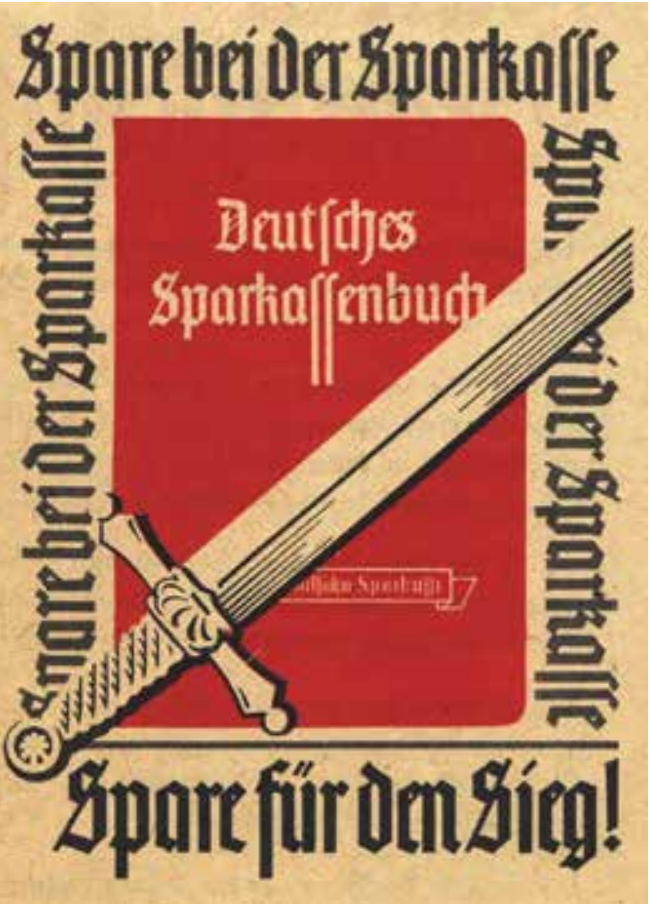


Reichswirtschaftsminister den Erwerb fördern. Der Sparkassenverband wies auf die Zeichnung dieser Li-Anleihen als „nationale Pflicht“ hin. Die Sparkassen beteiligten sich tatkräftig an der Konsolidierung von Schulden des Reichs, indem sie Anleihen und Schatzanweisungen zeichneten. ${ }^{47}$ Dadurch trugen sie zur Finanzierung der Aufrüstung bei. Die Kriegsfinanzierung erfolgte ebenfalls auf diese Weise, anstatt die Bevölkerung wie im Ersten Weltkrieg direkt durch Staatsanleihenkauf zu involvieren. ${ }^{48}$ Auch weil sie wegen einer staatlichen Bausperre kaum Geld im klassischen Hypothekengeschäft unterbringen konnten, suchten die sächsischen Sparkassen Alternativen. So kam es, dass schon 1940 mehr Geld in Wertpapieren als in Hypothekenkrediten angelegt war. ${ }^{49}$ Unter der NS-Herrschaft wurde mehrfach lenkend in die Anlagepolitik der Geldinstitute eingegriffen, auch um Sparkassenmittel für Anleihen nutzen zu können. Der Wegfall des privaten Wohnungsbaus vor dem Hintergrund der staatlichen Rohstoffbewirtschaftung trug zum entscheidenden Bedeutungsverlust der Sparkassenhypotheken bei..$^{50}$ Überliefert ist für Sachsen ein Bestand von 762 Millionen Reichsmark zum Kriegsende. Der Wertpapierbestand lag bei drei Milliarden Reichsmark. ${ }^{51}$ Aber nicht nur bei den Sparkassen, auch bei anderen Kreditinstituten, den Versicherungen und der Reichsbank hatte sich der Staat massiv verschuldet.

\section{Der Umgang mit dem Vermögen jüdischer Kunden}

Das Vermögen der von ihm verfolgten Juden verwendete er ebenfalls für seine Zwecke. Die Sparkassen wurden im „Dritten Reich“ bei den Maßnahmen gegen jüdischen Besitz einbezogen und die staatlichen Anordnungen dazu über den Deutschen Sparkassen- und Giroverband an die Regionalverbände weitergegeben..$^{52}$ Die Ausplünderung wurde rechtlich legitimiert. Die Steuerund Devisengesetzgebung stellte dabei ein wichtiges Instrument dar. So wurde zum Beispiel die noch aus der Weimarer Republik stammende Reichsfluchtsteuer missbraucht, um Auswanderer zu berauben und zu kriminalisieren. ${ }^{53}$ Wer die Steuer umging, wurde steckbrieflich gesucht. Das Vermögen wurde beschlagnahmt. Die Finanzbehörden gingen immer mehr mit Sicherungsbescheiden vor, um die Zahlung schon vor der Ausreise zu erzwingen. ${ }^{54}$ Die Steuer brachte dem Reich, das die Aufrüstungspolitik finanzieren musste, viel Geld ein. 1937 empfahl Heinrich Himmler, Steuer- und Devisenrechtsverstößen jüdischer Flüchtlinge grundsätzlich die Ausbürgerung wegen „volksschädlichen Verhaltens“ folgen zu lassen. ${ }^{55}$ Dies führte gemäß dem Gesetz über den Widerruf von Einbürgerungen und die Aberkennung der deutschen Staatsangehörigkeit vom 14. Juli 1933 automatisch zur Vermögensbeschlagnahmung. Das Gesetz, in den ersten Jahren des „Dritten Reichs“ vorwiegend gegen politische

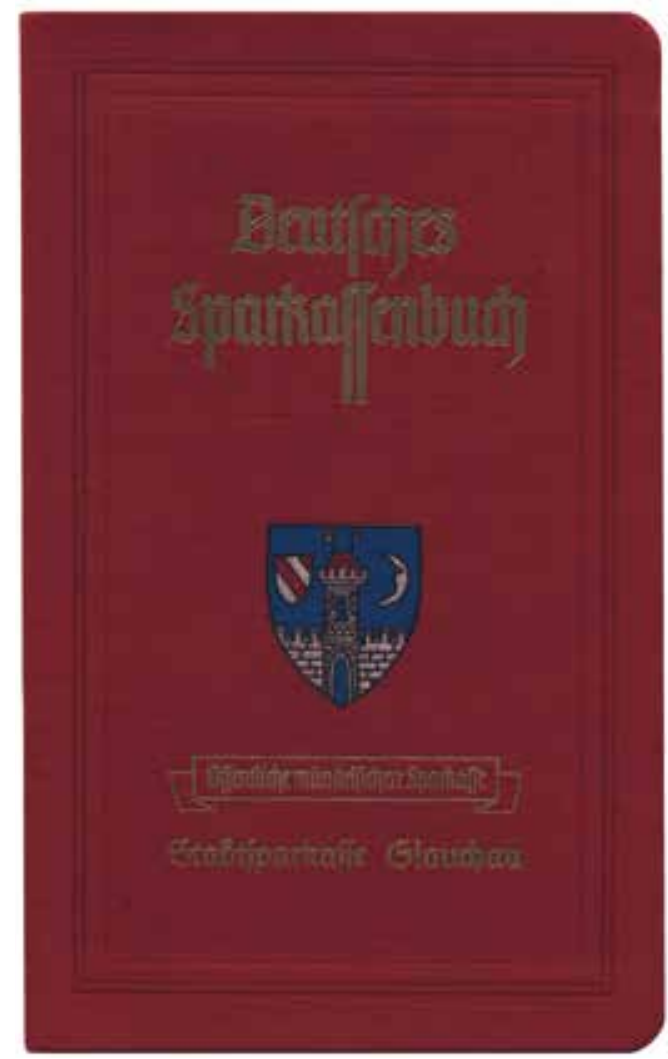

Emigranten genutzt, fungierte nun verstärkt als Instrument zur Judenverfolgung. ${ }^{56}$ Es folgten finanziell motivierte Massenausbürgerungen.

Auch die sächsischen Spar- und Girokassen wurden eingebunden. Sie durften an Personen, die von Steuersteckbriefen betroffen waren, keine Zahlungen leisten. Dem zuständigen Finanzamt, das die Beschlagnahme verfügte, hatten sie die dem Kunden zustehenden Forderungen oder sonstige Ansprüche mitzuteilen. ${ }^{57}$ Steuersteckbriefe wurden zur Information in der Deutschen Sparkassenzeitung abgedruckt. Es konnten auch Namenslisten beim DSGV bestellt werden. Eine Recherche für den Zeitraum von 1933 bis 1939 ergab, dass auch ehemals vor allem in Dresden, Leipzig und Chemnitz wohnhafte jüdische Emigranten steckbrieflich gesucht wurden. Was die Folgen der Ausbürgerungen betraf, so versendeten der Sparkassenverband und der Giroverband in Sachsen von Februar 1937 bis März 1939 spezielle Rundschreiben an die Mitglieder. Diese enthielten die bereits im Reichsanzeiger veröffentlichten Daten. Auch die kommunalen Kreditinstitute hatten Feststellungen über eventuelle Guthaben zu treffen und diese Informationen weiterzuleiten. ${ }^{58}$ Die Finanzbehörden waren für die folgenden Beschlagnahmungen und die Verwertung des Besitzes zu Gunsten des Reichs zuständig. Die fiskalisch motivierte Ausweitung der Ausbürgerungspolitik spiegelt sich in den Rundschreiben wider. Die Namenslisten, darunter auch emigrierte Juden insbesondere aus Leipzig, Dresden und Chemnitz, wurden immer länger. Ab Januar 1939 übermittelten die Verbände auch Daten von Familienangehörigen, die ebenfalls die
1943 gab es mehr

Sparkassenbücher als Einwohner in Sachsen.

( ) Historisches Archiv des

Ostdeutschen Sparkassenverbandes

48 Vgl. Pohl/Rudolph/Schulz 2005 (wie Anm. 11), S. 203.

49 Vgl. Niederschrift über die Vorstandssitzung vom 13. Juni 1940 sowie Geschäftsbericht des Sächsischen Sparkassenverbandes und des Giroverbandes Sächsischer Gemeinden für 1940, S.21, OSV Archiv.

50 Vgl. Pohl/Rudolph/Schulz 2005 (wie Anm. 11), S. 215.

51 Vgl. Bericht des Sächsischen Sparkassenverbandes vom 10. August 1948, OSV Archiv.

52 Vgl. Pohl/Rudolph/Schulz 2005 (wie Anm. 11), S. S. 175.

53 Vgl. Martin Friedenberger: Fiskalische Ausplünderung. Die Berliner Steuer- und Finanzverwaltung und die jüdische Bevölkerung 19331945. Berlin 2008, S. 67 ff.

54 Vgl. Christiane Kuller: Bürokratie und Verbrechen. Antisemitische Finanzpolitik und Verwaltungspraxis im nationalsozialistischen Deutschland. München 2013, S. 198.

55 Vgl. Christoph Franke: Die Rolle der Devisenstellen bei der Enteignung der Juden. In: Katharina Stengel (Hrsg.): Vor der Vernichtung. Die staatliche Enteignung der Juden im Nationalsozialismus. Frankfurt/New York 2007, S. 85.

56 Vgl. Friedenberger 2008 (wie Anm. 53), S. 246.

57 Vgl. Verbandsrundschreiben vom 21. März 1938, OSV Archiv.

58 Vgl. Verbandsrundschreiben vom 15. Februar 1937, OSV Archiv.

59 Vgl. Verbandsrundschreiben vom 23. Februar 1939, OSV Archiv.

60 Vgl. Verbandsrundschreiben vom 5. Juli 1939, OSV Archiv.

61 Vgl. Verbandsrundschreiben vom 29. Juli 1938 sowie 3. November 1938, OSV Archiv.

62 Ralf Banken: Das nationalsozialistische Devisenrecht als Steuerungs- und Diskriminierungsinstrument 1933-1945. In: Johannes Bähr/Ralf Banken (Hrsg.): Wirtschaftssteuerung durch Recht im Nationalsozialismus. Studien zur Entwicklung des Wirtschaftsrechts im Interventionsstaat des „Dritten Reiches“. Frankfurt am Main 2006, S. 214. 

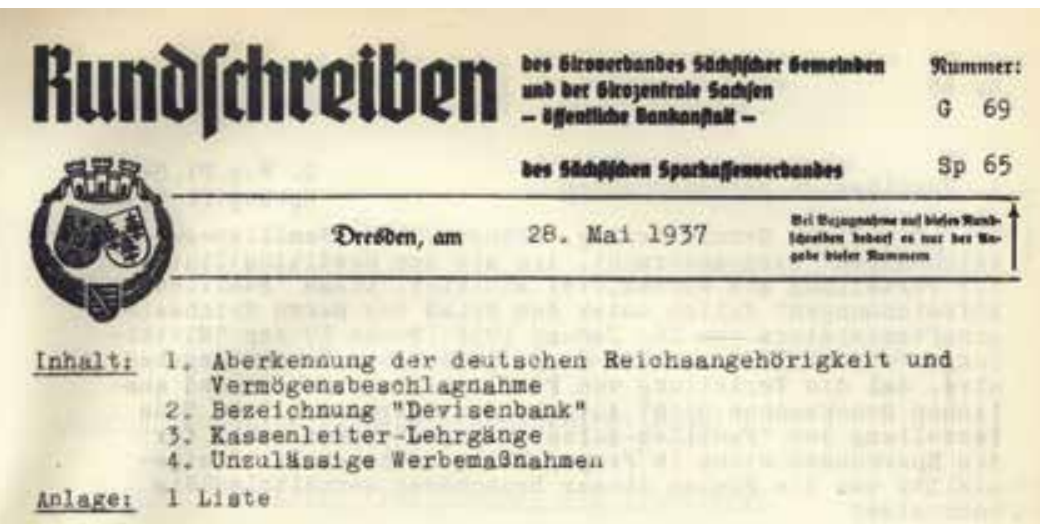

1. Aberkenuang der deutgohen Reichsangehorizbeis und vermogensbesohiagnahme

a. Reg.P1. 8497 Sp.Reg.P1: 8597

Mit dem Rundschreiben volm 15. Pebruar 1937 hacea wir eine L1ste derjenigen Peraonen ubersandt, denen die deutsche staataangehbrigkeit sberikannt und deren Vermbgen beschlagnahat worden 1st. Diese Liste wird duroh die Anlage erganzt. Wir weisen erneut daraup hin, das die Kreditinatitute - also auch die sparkassen und Girokassen nachzuprufen haben, ob aie fur die betreffenden Peraonen Vermógensnachzuprufen haben, ob ale fur die betreffenden Personen Vermogens
werte verwalten. Solite das der Pall sein, so sind die Konten sofort zu gperren und $\mathrm{zu}$ melden. Die Meldung ist uns zur Feiterleitung an die in Prage komande stelle zu ubersenden.

Rundschreiben vom 28. Mai 1937 mit der Mitteilung der Vermögensbeschlagnahme

- Historisches Archiv des

Ostdeutschen Sparkassenverbandes

63 Vgl. Friedenberger 2008 (wie Anm. 53), S. $257 \mathrm{ff}$

64 Vgl. Müller 2003 (wie Anm. 8), S. 301.

65 Vgl. Verbandsmitteilung vom 23. Februar 1939, OSV Archiv.

66 Vgl. Verbandsrundschreiben vom 5. Januar 1942, OSV Archiv.

67 Verbandsrundschreiben vom 25. Juni 1942, OSV Archiv.

68 Mitteilungsblatt der Sächsischen Sparkassen- und Giroorganisation vom 15 . Dezember 1944, OSV Archiv.

Autor

Thomas Einert, M.A.

Historisches Archiv des Ostdeutschen Sparkassenverbandes

Leipziger Straße 51, 10117 Berlin thomas.einert@osv-online.de deutsche Staatsangehörigkeit verloren. Das jüngste Kind, ein jüdischer Junge aus Chemnitz, war noch nicht einmal sechs Jahre alt. ${ }^{59}$ Für die Kassen gab es den Hinweis, dass auch nachträglich festgestelltes Vermögen beschlagnahmt werde. Besondere Fälle waren dem Reichsführer SS und Chef der deutschen Polizei mitzuteilen. Weil die Unterlagen der Gestapo, die zur Erstellung der Rundschreiben genutzt wurden, immer umfangreicher ausfielen, erfolgte die Einstellung des Versands. Zur Prüfung der Konten und Depots gab es fortan die Listen der Staatspolizei direkt per Umlauf. ${ }^{60}$

Bereits 1938 mussten die sächsische Spar- und Girokassen nach Vorgaben staatlicher Zollfahndungsstellen jüdische Schließfächer und Konten ermitteln und gemäß Sicherungsanordnungen Verfügungsbeschränkungen einführen, um eine Kapitalflucht zu verhindern. ${ }^{61}$ Der Staat bestimmte, dass Juden nur noch in begrenztem Umfang über ihre Einlagen verfügen durften. Tatsächlich waren Auswanderungswillige schon vor der Pogromnacht „vermögenstechnisch [...] vollkommen gefangen" ${ }^{62}$. Auch bereits ausgewanderte Juden hatten die dann eingeführte „Judenvermögensabgabe“ zu zahlen, verloren sonst die Staatsangehörigkeit und damit ihr Vermögen in Deutschland. ${ }^{63}$ Die kollektive Ausbürgerung und Enteignung machte die Einzelverfahren dazu obsolet. Die Nationalsozialisten schufen mit der 11. Verordnung zum Reichsbürgergesetz vom 25. November 1941 die Legitimation, alle im Ausland befindlichen deutschen Juden, die keine neue Staatsangehörigkeit hatten, komplett $\mathrm{zu}$ berauben. Die Verschleppung der noch im Reich befindlichen Menschen wurde mit der Verlegung des Wohnsitzes ins Ausland gleichgesetzt. Im Hinblick auf die Zielorte der einsetzenden Massendeportationen galten die von
Deutschland besetzten und verwalteten Gebiete, unter anderem das „Generalgouvernement“, als Ausland. ${ }^{64}$

1939 waren die sächsischen Spar- und Girokassen angewiesen worden, Konten und Depots durch Eintragung der gesetzlich vorgegebenen Zwangsnamen „Israel“ und „Sara“ zu kennzeichnen. ${ }^{65}$ Das Reichsfinanzministerium hatte im selben Jahr festgelegt, dass Juden nur noch 300 Reichsmark monatlich ohne Genehmigung von ihren „Sicherungskonten“ ausgezahlt bekamen. In Zusammenhang mit der Verordnung zum Reichsbürgergesetz erfolgte 1941 ein Verfügungsverbot der Gestapo, das auch Konten betraf. Der Zugriff war nur noch in Ausnahmefällen möglich und der Auszahlungsbetrag auf 150 Reichsmark herabgesetzt. Darüber wurden alle Kassen Anfang Januar 1942 informiert. ${ }^{66}$ Das Verbandsrundschreiben thematisierte auch den Vermögensverfall bei Ausbürgerungen. So sollten jüdische Kunden den Nachweis erbringen, dass oben angesprochene Verordnung für sie nicht galt. Bis dahin blieben Konten und Depots gesperrt. Auch wenn nur ein gewisser Verdacht bestand, dass es sich um jüdischen Besitz handelte, erfolgte eine vorläufige Sperre. Weil die Identifizierung Emigrierter anhand des Namens Probleme bereiten konnte, wurde bald empfohlen, in zweifelhaften Fällen den Chef der Sicherheitspolizei und des SD die Feststellung treffen zu lassen. Über die in den Osten „abgeschobenen“ Juden informierten die Oberfinanzpräsidenten der Bezirke. Die Vermögenswerte mussten dann abgeliefert werden. Anzumerken ist, dass solche speziellen Hinweise nur diejenigen sächsischen Sparkassen und Girokassen erhielten, die ihren Verband über jüdische Kunden informiert hatten. Das letzte offizielle Rundschreiben datiert zum 25. Juni 1942. In ihrem Interesse, um sich vor etwaigen Schwierigkeiten zu schützen, sollten die Kassen gewissenhaft prüfen und Meldung $\mathrm{zu}$ noch bestehenden Konten machen. Auch Fehlanzeigen wurden gefordert. Dabei sei egal, „ob sich die jüdischen Kontoinhaber noch im Inland oder im Ausland befinden und ob sie ausgewandert oder abgeschoben oder verstorben sind "67. Ein Jahr später erfolgte eine weitere und letzte Verordnung zum Reichsbürgergesetz. Nach dem Tode eines Juden in Deutschland sollte sein Vermögen automatisch dem Reich verfallen. Man wollte dadurch zum Beispiel ans Geld der Menschen kommen, die sich vor der Deportation das Leben nahmen. Diese 13. Verordnung bildete den gesetzlichen Abschluss der legalisierten Ausplünderung der Juden. Eine Durchführungsverordnung bestimmte am 1 . September 1944, dass die Regelung nur tote deutsche und staatenlose Juden betraf, was der Sächsische Sparkassen- und Giroverband weiterleitete. „Damit ist die früher ausgesprochene Empfehlung, auch Konten von verstorben Juden ausländischer Staatsangehörigkeit vorsorglich zu sperren, erledigt.““ ${ }^{\circ}$ 